Research Paper

\title{
The Application of Dehydroepiandrosterone on Improving Mitochondrial Function and Reducing Apoptosis of Cumulus Cells in Poor Ovarian Responders
}

\author{
Li-Te Lin 1, 2, 3, Peng-Hui Wang 3, 4, 5, 6, 7, Zhi-Hong Wen ${ }^{8}$, Chia-Jung Li' ${ }^{9}$, San-Nung Chen², Eing-Mei Tsai10, 11, \\ Jiin-Tsuey Cheng ${ }^{1 凶}$, Kuan-Hao Tsui1 ${ }^{1,2,3,12 \bowtie}$
}

1. Department of Biological Science, National Sun Yat-sen University, Kaohsiung, Taiwan;

2. Department of Obstetrics and Gynecology, Kaohsiung Veterans General Hospital, Kaohsiung, Taiwan;

3. Department of Obstetrics and Gynecology, National Yang-Ming University School of Medicine, Taipei, Taiwan;

4. Department of Obstetrics and Gynecology, Taipei Veterans General Hospital, Taipei, Taiwan;

5. Department of Obstetrics and Gynecology, National Yang-Ming University Hospital, Ilan, Taiwan;

6. Immunology Center, Taipei Veterans General Hospital, Taipei, Taiwan;

7. Department of Medical Research, China Medical University Hospital, Taichung, Taiwan;

8. Department of Marine Biotechnology and Resources, National Sun Yat-sen University, Kaohsiung, Taiwan;

9. Institute of Clinical Medicine, College of Medicine, National Cheng Kung University, Taiwan;

10. Department of Obstetrics and Gynecology, Kaohsiung Medical University Hospital, Kaohsiung, Taiwan ;

11. Graduate Institute of Medicine, College of Medicine, Kaohsiung Medical University, Kaohsiung, Taiwan;

12. Department of Pharmacy and Master Program, College of Pharmacy and Health Care, Tajen University, Pingtung County, Taiwan.

$\square$ Corresponding authors: Kuan-Hao Tsui, M.D., Ph.D., Department of Obstetrics and Gynecology, Kaohsiung Veterans General Hospital, Kaohsiung, Taiwan Mailing address: No.386, Dazhong 1st Rd., Zuoying Dist., Kaohsiung City 81362, Taiwan Phone: +886-7-3422121 ext 4014; Fax: +886-7-3468189; E-mail: khtsui60@gmail.com. Jiin-Tsuey Cheng, Ph.D., Department of Biological Science, National SunYat-sen University, Kaohsiung, Taiwan Mailing address: 70 Lienhai Rd., Kaohsiung 80424, Taiwan Phone: +886-7-5252000 ext 3624; Fax: +886-7-5253624; E-mail: tusya@mail.nsysu.edu.tw

( $)$ Ivyspring International Publisher. This is an open access article distributed under the terms of the Creative Commons Attribution (CC BY-NC) license (https://creativecommons.org/licenses/by-nc/4.0/). See http://ivyspring.com/terms for full terms and conditions.

Received: 2016.12.12; Accepted: 2017.04.01; Published: 2017.05.13

\begin{abstract}
Poor ovarian responders (PORs) pose a great challenge for in vitro fertilization (IVF). Previous studies have suggested that dehydroepiandrosterone (DHEA) may improve IVF outcomes in PORs. The current study attempted to investigate the clinical benefits of DHEA in PORs and the possible mechanisms of DHEA on cumulus cells (CCs). This was a prospective study performed at one tertiary center from January 2015 to March 2016. A total of 131 women who underwent IVF treatment participated, including 59 normal ovarian responders (NORs) and 72 PORs. PORs were assigned to receive DHEA supplementation or not before the IVF cycle. For all patients, CCs were obtained after oocyte retrieval. In the CCs, mRNA expression of apoptosis-related genes and mitochondrial transcription factor A (TFAM) gene, terminal deoxynucleotidyl transferase dUTP nick end labeling assay, mitochondrial dehydrogenase activity and mitochondrial mass were measured. The results indicated that PORs with DHEA supplementation produces a great number of top-quality embryos at day 3 and increased the number of transferred embryos and fertilization rate compared with those without DHEA supplementation. Additionally, supplementation with DHEA in PORs decreased DNA damage and apoptosis in CCs while enhancing the mitochondrial mass, mitochondrial dehydrogenase activity and TFAM expression in CCs. In conclusion, our results showed that the benefits of DHEA supplementation on IVF outcomes in PORs were significant, and the effects may be partially mediated by improving mitochondrial function and reducing apoptosis in CCs.
\end{abstract}

Key words: apoptosis; cumulus cells; dehydroepiandrosterone; mitochondria; poor ovarian responders. 


\section{Introduction}

Poor ovarian responders (PORs), characterized by a poor response to controlled ovarian stimulation (COS), pose a great obstacle for in vitro fertilization (IVF). PORs yield poor oocyte quality and ovarian reserve, leading to extremely low live birth rates $[1,2]$. Several strategies, including variety of COS protocols [3] and various adjuvant supplements [4-6], were proposed as an attempt to improve the reproductive outcomes in PORs. Regarding adjuvant supplements, dehydroepiandrosterone (DHEA) was considered a potential agent to better clinical outcomes in PORs [7, 8]. DHEA, an endogenous steroid produced by the zona reticularis of the adrenal glands and by ovarian theca cells, is a precursor to estradiol and testosterone [9]. The dehydroepiandrosterone sulfate level in follicular fluid has been reported to be a predictor of oocyte maturation, fertilization, embryo development and live birth in women undergoing IVF cycles [10]. The Cochrane review concluded that pre-treatment with DHEA may be associated with improved live birth rates in PORs undergoing IVF cycles [11]. However, the possible mechanisms of improved IVF outcomes in PORs following DHEA treatment are not fully known.

Oocytes were protected and nurtured from surrounding somatic cells, including cumulus cells (CCs) and granulosa cells [12]. CCs and oocytes form cumulus-oocyte complex (COC), which communicate with each other through specialized gap junctions [12, 13]. Numerous studies indicate that gene expression in CCs can serve as biomarkers for oocyte or embryo quality and pregnancy outcomes [14-16]. Our previous self-controlled studies demonstrated the potential anti-apoptotic effect on CCs following DHEA treatment $[17,18]$. Apoptosis plays a critical role on oogenesis, folliculogenesis, oocyte loss, selection, atresia and luteogenesis [19]. Several studies showed that the apoptosis of CCs was associated with impaired oocyte maturation, fertilization, embryo growth and pregnancy outcomes [20-23].

Mitochondria plays an important role on the intrinsic apoptosis pathway mediated by the BCL2 family [24]. When stress stimuli are transduced to mitochondria, $B A X$ and $B A K$, pro-apoptotic members of the BCL2 family, increase the mitochondrial membrane permeability to proteins such as cytochrome c, leading to caspase cascade activation [25]. Our previous self-controlled study revealed that mitochondrial dehydrogenase activity of CCs significantly increased in PORs after DHEA supplementation. Mitochondria are involved in oocyte growth and embryo development; interference with mitochondrial function contributes to arrest of oocyte maturation, impaired fertilization and compromised embryo development [26-28].

Based on our previous studies $[17,18]$ and the numerous studies discussed thus far, we hypothesized that DHEA may improve reproductive outcomes in PORs through increasing mitochondrial function and decreasing apoptosis in CCs. To verify this hypothesis, we compared the IVF outcomes of normal ovarian responders (NORs), PORs with or without DHEA supplementation and collected their CCs for researches. In fact, this study was a further research of our previous work [18] by enrolling more patients, adding control groups and performing more experiments.

\section{Materials and Methods}

\section{Patients and design}

This prospective study was performed at the Reproductive Center of the Kaohsiung Veterans General Hospital between January 2015 and March 2016. The study enrolled NORs and PORs. The inclusive criteria for NORs included the following: (1) antral follicle counts (AFC) $\geq 5$ or anti-Müllerian hormone $(\mathrm{AMH}) \geq 1 \mathrm{ng} / \mathrm{mL}$ and (2) the number of retrieved oocytes was between 5 and 15. PORs met the Bologna criteria [29], having at least two of the three following features: (1) advanced maternal age $(\geq 40$ years) or any other risk factor for POR, (2) a previous POR ( $\leq 3$ oocytes with a conventional stimulation protocol), and (3) an abnormal ovarian reserve test. An abnormal ovarian reserve test was defined as $\mathrm{AFC}<5$ or $\mathrm{AMH}<1 \mathrm{ng} / \mathrm{mL}$ in this study. Moreover, two episodes of a previous POR after maximal stimulation alone would be sufficient to define a patient as a POR. PORs were divided into 2 groups in this study. In the POR group, patients directly underwent an IVF cycle without DHEA pre-treatment. In the POR/DHEA group, patients received DHEA supplementation (CPH; Formulation Technology, Oakdale, CA, USA) of $90 \mathrm{mg}$ per day at least 2 months (8 to 16 weeks, mean 12.6 weeks) before entering an IVF cycle.

\section{Ethical approval}

All procedures performed in studies involving human participants were in accordance with the ethical standards of the institutional and/or national research committee and with the 1964 Helsinki declaration and its later amendments or comparable ethical standard. The institutional review board at Kaohsiung Veterans General Hospital approved all study procedures. The study was performed in accordance with approved guidelines. Informed 
consent was obtained from all individual participants included in the study.

\section{Treatment protocol}

All participants underwent a gonadotropinreleasing hormone $(\mathrm{GnRH})$ antagonist protocol, as previously described [18]. Briefly, ovarian stimulation occurred on day 2 of the menstrual cycle with daily recombinant follicle-stimulating hormone $(\mathrm{rFSH}) \pm$ recombinant luteinizing hormone $(\mathrm{rLH})$, including Gonal-F (rFSH, Merck KGaA, Darmstadt, Germany), Pergovaris (rFSH + rLH, Merck Serono, Aubonne, Switzerland) or Merional ( $\mathrm{rFSH}+\mathrm{rLH}$, Institut Biochimique SA, Lamone, Switzerland). A GnRH antagonist (Cetrotide $0.25 \mathrm{mg}$; Merck Serono, Idron, France) was administered when the leading follicle reached a diameter of 12-14 mm. Recombinant human chorionic gonadotropin (Ovidrel, Merck Serono, Modugno, Italy) was administered until at least three dominant follicles reached $\geq 17 \mathrm{~mm}$ in diameter in the NORs or at least one dominant follicle reached $\geq 17 \mathrm{~mm}$ in diameter in the PORs. Transvaginal oocyte retrieval was performed $34-36 \mathrm{~h}$ later. Intracytoplasmic sperm injection (ICSI) was conducted in all PORs to reduce the possibility of fertilization failure. NORs underwent ICSI in the cases of poor sperm quality. Embryos were assessed and scored according to the criteria established by the Istanbul consensus workshop [30]. Embryo transfer was done under transabdominal sonographic guidance on day 3 after oocyte retrieval in the PORs and on day 3 or day 5 after oocyte retrieval, depending on the embryo status in the NORs.

Luteal phase support was started on the day of oocyte retrieval. Daily progesterone, including Crinone 8\% gel (Merck Serono, Hertfordshire, UK) and Duphaston $4 \mathrm{mg}$ (Abbott, Olst, The Newtherlands) were given routinely. A pregnancy test was performed 14 days later. Once a positive pregnancy test was observed, progesterone was continued for an additional 6 weeks. Clinical pregnancy was established if visualization of a fetal heart beat was found in an intrauterine gestational sac by transvaginal ultrasound. Ongoing pregnancy was determined by the presence of a fetal heart beat beyond 20 weeks of gestation. Live birth was defined as delivery of a live fetus after 20 completed weeks of gestation.

\section{Cumulus-oocyte complex grade and cumulus cell collection}

After oocyte retrieval, COCs were collected, washed, and visually classified into one of three groups based on the degree of oocyte and cumulus expansion, as previously described [31]. COCs were incubated in the IVF medium covered with paraffin oil until denudation. $2 \mathrm{~h}$ later, COCs were denuded individually using hyaluronidase (SynVitro $^{\text {TM }}$ Hyadase, Origo, Målov Denmark, Knardrupvej) for $30 \mathrm{~s}$ at most. CCs were isolated, pooled per patient, and transferred to a $15-\mathrm{mL}$ centrifugation tube containing $4 \mathrm{~mL}$ of Histopaque 1077 (Sigma Chemical, St. Louis, MO, USA). CCs were separated from red blood cells by centrifugation at $600 \mathrm{~g}$ for $10 \mathrm{~min}$. CCs formed a thin layer between the Histopaque and the medium. Cells were removed and placed in a new centrifugation tube and washed using IVF medium, with centrifugation at $600 \mathrm{~g}$ for 10 minutes. The supernatant was discharged and the CCs were placed at $-80^{\circ} \mathrm{C}$ for further study.

\section{RNA isolation and quantitative real-time polymerase chain reaction (Q-PCR)}

The method for Q-PCR was as described previously [17]. Total RNA was extracted from tissue specimens using the acid-phenol guanidium method. Briefly, TRIzol was added to the CCs. The mixture was pipetted to mix and allowed to sit for $5 \mathrm{~min}$ at room temperature. Chloroform was added, mixed, and allowed to incubate at room temperature for 10 $\mathrm{min}$. The mixture was centrifuged at $12,000 \mathrm{~g}$ for 20 min, and the supernatant was transferred to a fresh tube. Isopropanol was added, mixed, and incubated for $10 \mathrm{~min}$ at room temperature. The solution was centrifuged at 12,000 $\mathrm{g}$ for $30 \mathrm{~min}$, and the RNA was purified as above. The pellet was washed twice with $70 \%$ ethanol, re-suspended in diethylpyrocarbonate (DEPC)-treated water, and stored at $-80^{\circ} \mathrm{C}$. All Q-PCRs were performed using an ABI Prism 7700 Sequence Detection System (Perkin-Elmer Applied Biosystems, Foster City, CA, USA). PCR was performed using the SYBR Green PCR Core Reagents kit (Perkin-Elmer Applied Biosystems). The thermal cycling conditions included an initial denaturation step at $95^{\circ} \mathrm{C}$ for $10 \mathrm{~min}$, and 40 cycles at $95^{\circ} \mathrm{C}$ for $15 \mathrm{~s}$, and $60^{\circ} \mathrm{C}$ for $1 \mathrm{~min}$. Specific PCR amplification products were detected by the fluorescent double-stranded DNA-binding dye, SYBR Green. Experiments were performed with triplicates for each data point. All samples with a coefficient of variation for $\mathrm{Ct}$ value $>1 \%$ were retested. The primers used for Q-PCR analysis are shown in supplemental Table S1.

\section{Terminal deoxynucleotidyl transferase dUTP nick end labeling (TUNEL) analysis}

After oocyte denudation, CCs were isolated and washed with phosphate-buffered saline (PBS) and fixed with $4 \%$ formaldehyde in PBS ( $\mathrm{pH} 7.4$ ) for $20 \mathrm{~min}$ at $4{ }^{\circ} \mathrm{C}$. TUNEL analysis with fluorescein was performed with the ApopTag Fluorescein Direct In 
Situ Apoptosis Detection Kit (Millipore Co.). Slides were counterstained with 4',6-diamidino-2-phenylindole (DAPI) for $10 \mathrm{~min}$, dehydrated, cleared, and cover-slipped.

\section{Mitochondrial dehydrogenase activity assay}

Mitochondrial dehydrogenase activity was analyzed using the cell counting kit-8 (CCK-8, Enzo Life Sciences Inc., NY, USA) that detected the metabolic activity of the cells. CCK-8 reagent $(10 \mu \mathrm{L})$ was added to each well, and the cells were then incubated at $37^{\circ} \mathrm{C}$ for $4 \mathrm{~h}$. Absorbance was recorded using an enzyme-linked immunosorbent assay microplate reader at $450 \mathrm{~nm}$.

\section{Mitochondrial mass measurement}

The collected CCs were washed twice in PBS by centrifugation to avoid blood contamination, and the pellet was resuspended in PBS for analysis using an image flow cytometer. For the image cytometry assay, the cell pellet was gently and thoroughly re-suspended in the remaining $20 \mu \mathrm{L}$ of media, and 20 $\mu \mathrm{L}$ was transferred to the disposable counting slide. Bright-field and fluorescent images of the sample were captured using the filter optics module VB-535-402 for MitoTracker green detection at an exposure time of $1 \mathrm{~s}$ with a size cut-off of $0.1 \mu \mathrm{m}$.

\section{Statistical analysis}

Standard statistical procedures were carried out using the Statistical Package for Social Sciences (SPSS) version 20.0. Normality of quantitative variables was revealed by Kolmogorov-Smirnov test. One-way analysis of variance (ANOVA) was used in comparing quantitative variables and Bonferroni's test was applied for post-hot test. The categorical variables were compared using the chi-square test. Data are presented as the mean \pm the standard deviation (SD) of three biological replicates. Comparisons with a $p$ value $<0.05$ were considered significant.

\section{Results}

\section{Basic characteristics of patients undergoing IVF cycles}

The basic characteristics among the NOR, POR and POR/DHEA groups are presented in Table 1. A total of 131 women who underwent IVF treatment were enrolled, including 59 NORs and 72 PORs. Among the PORs, 34 women were treated with DHEA (POR/DHEA group) and 38 women were not (POR group). The mean age was significantly lower in the NOR group (35.9 years) than in the POR (39.4 years) and POR/DHEA groups (39.3 years). However, there were no significant differences among the three groups regarding body mass index or the percentage of primary infertility and secondary infertility. Infertility duration was significantly longer in the POR group (6.3 years) than in the NOR group (3.6 years). Furthermore, women in the POR/DHEA group had a higher percentage of multiple previous failed IVF cycles ( $\geq 3$ times) than those in the NOR group (47.1\% in the POR/DHEA group vs. $8.5 \%$ in the NOR group, $p<0.05)$.

Table 1. Basic characteristics of patients in the NOR, POR and POR/DHEA groups

\begin{tabular}{lccc}
\hline Parameters & NOR $(\mathrm{n}=59)$ & POR $(\mathrm{n}=38)$ & $\begin{array}{l}\text { POR/DHEA } \\
(\mathrm{n}=34)\end{array}$ \\
\hline Age (years) & $35.9 \pm 3.9$ & $39.4 \pm 3.5^{\mathrm{a}}$ & $39.3 \pm 2.4^{\mathrm{a}}$ \\
Body mass index $(\mathrm{kg} / \mathrm{m} 2)$ & $22.0 \pm 3.6$ & $21.1 \pm 3.8$ & $21.1 \pm 2.0$ \\
Infertility duration (year) & $3.6 \pm 3.0$ & $6.3 \pm 5.2^{\mathrm{a}}$ & $5.4 \pm 3.9$ \\
Types of infertility n (\%) & & & \\
Primary infertility & $22(37.3)$ & $19(50.0)$ & $16(47.1)$ \\
Secondary infertility & $37(62.7)$ & $19(50.0)$ & $18(52.9)$ \\
Basal FSH (IU/l) & $4.3 \pm 1.8$ & $7.1 \pm 5.4^{\mathrm{a}}$ & $6.4 \pm 2.8^{\mathrm{a}}$ \\
Antral follicle counts (n) & $10.7 \pm 3.4$ & $3.5 \pm 1.4^{\mathrm{a}}$ & $3.3 \pm 1.1^{\mathrm{a}}$ \\
Anti-Müllerian hormone (ng/ml) & $3.7 \pm 2.0$ & $1.0 \pm 0.6^{\mathrm{a}}$ & $1.0 \pm 1.2^{\mathrm{a}}$ \\
Previous IVF failure $\mathrm{n}(\%)$ & & & \\
0 & $34(57.6)$ & $14(36.8)$ & $8(23.5)^{\mathrm{a}}$ \\
1-2 & $20(33.9)$ & $14(36.8)$ & $10(29.4)$ \\
$\geq 3$ & $5(8.5)$ & $10(26.3)$ & $16(47.1)^{\mathrm{a}}$ \\
\hline
\end{tabular}

NOR: normal ovarian responder; POR: poor ovarian responder; DHEA: dehydroepiandrosterone; FSH: follicle stimulation hormone; IVF: in vitro fertilization

aSignificant difference compare with NOR group, $p<0.05$.

As expected, the mean number of AFC and serum AMH levels were markedly higher in the NOR group than in the POR and POR/DHEA groups. Moreover, the mean FSH level was significantly lower in the NOR group than in the POR and POR/DHEA groups.

\section{Cycle characteristics and pregnancy outcome of patients undergoing IVF cycles}

The cycle characteristics and pregnancy outcome among the NOR, POR and POR/DHEA groups are shown in Table 2. There were no significant differences among the three groups in terms of stimulation duration and gonadotropin dose. However, the number of retrieved oocytes, metaphase II oocytes, top-quality embryos at day 3 and transferred embryos were significantly higher in the NOR group than those in the POR and POR/DHEA groups. Similarly, the clinical pregnancy rate, ongoing pregnancy rate and live birth rate were markedly greater in the NOR group than in the POR and POR/DHEA groups.

When comparing the POR/DHEA group with the POR group, the number of top-quality embryos at day 3 (1.2 \pm 1.1 vs. $0.3 \pm 0.6$, respectively, $p<0.05)$, transferred embryos $(2.1 \pm 0.9$ vs. $1.1 \pm 1$, respectively, $p<0.05)$ and fertilization rate $(75.9 \%$ vs. $58.8 \%$, 
respectively, $p<0.05$ ) were significantly greater. Moreover, the POR/DHEA group was associated with a potentially greater number of retrieved oocytes ( $3.5 \pm 2$ vs. $2.3 \pm 1.2$, respectively) and metaphase II oocytes ( $2.2 \pm 1.6$ vs. $1.1 \pm 0.9$, respectively) compared with the POR group, but these differences were not significant. Similarly, the POR/DHEA group displayed higher clinical pregnancy rate $(18.7 \%$ vs. $5.2 \%$, respectively), ongoing pregnancy rate $(15.6 \%$ vs. $2.6 \%$, respectively) and live birth rate $(12.9 \%$ vs. $2.6 \%$, respectively) than the POR group. However, the difference was not statistical significant.

\section{Effects of DHEA supplementation on cumulus-oocyte complex grade}

As shown in Fig. 1A, DHEA supplementation ameliorated oocyte maturation and cumulus expansion. The CCs from the POR/DHEA group significantly increased proportion of grade 3 COC compared to those from the POR group $(58.6 \%$ vs. $28.4 \%$, respectively, $p<0.001$ ) (Fig. 1B). In addition, the mean COC grade was significantly greater in the POR/DHEA group than that in the POR group (2.51 \pm 0.64 vs. $1.97 \pm 0.78$, respectively, $p<0.001$ ) (Fig. 1C).

Table 2. Cycle characteristics and pregnancy outcome in the NOR, POR and POR/DHEA groups

\begin{tabular}{llll}
\hline Parameters & NOR $(\mathrm{n}=59)$ & POR $(\mathrm{n}=38)$ & $\begin{array}{l}\text { POR/DHEA } \\
(\mathrm{n}=34)\end{array}$ \\
\hline Stimulation duration (days) & $11.0 \pm 2.3$ & $10.2 \pm 2.2$ & $10.6 \pm 1.8$ \\
HMG/FSH dose (IU) & $3152.1 \pm$ & $2967.5 \pm$ & $3097.0 \pm 574.3$ \\
& 778.8 & 831.5 & \\
No. of oocytes retrieved (n) & $9.5 \pm 3.6$ & $2.3 \pm 1.2^{\mathrm{a}}$ & $3.5 \pm 2.0^{\mathrm{a}}$ \\
No. of metaphase II oocytes (n) & $5.4 \pm 2.6$ & $1.1 \pm 0.9 \mathrm{a}$ & $2.2 \pm 1.6^{\mathrm{a}}$ \\
No. of top-quality D3 embryos (n) & $2.4 \pm 1.8$ & $0.3 \pm 0.6^{\mathrm{a}}$ & $1.2 \pm 1.1^{\mathrm{a}, \mathrm{b}}$ \\
No. of embryos transfer (n) & $2.8 \pm 0.8$ & $1.1 \pm 1.0^{\mathrm{a}}$ & $2.1 \pm 0.9^{\mathrm{a}, \mathrm{b}}$ \\
Fertilization rate (\%) & 69.0 & 58.8 & $75.9 \mathrm{~b}$ \\
Clinical pregnancy rate \% (n) & $55.1(27 / 49)$ & $5.2^{\mathrm{a}}(2 / 38)$ & $18.7 \mathrm{a}(6 / 32)$ \\
Ongoing pregnancy rate \% (n) & $46.9(23 / 49)$ & $2.6^{\mathrm{a}}(1 / 38)$ & $15.6^{\mathrm{a}}(5 / 32)$ \\
Live birth rate \% (n) & $43.7(21 / 48)$ & $2.6^{\mathrm{a}}(1 / 38)$ & $12.9 \mathrm{a}(4 / 31)$ \\
\hline
\end{tabular}

NOR: normal ovarian responder; POR: poor ovarian responder; DHEA:

dehydroepiandrosterone; HMG: human menopausal gonadotrophin; FSH: follicle stimulation hormone; D: day

aSignificant difference compare with NOR group, $p<0.05$

bSignificant difference compare with POR group, $p<0.05$.

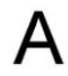

NOR
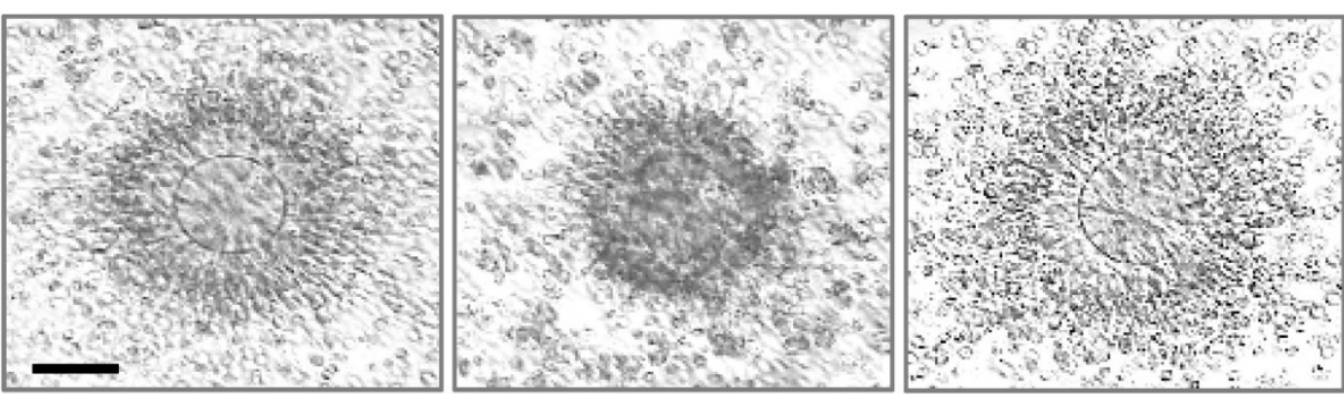

B

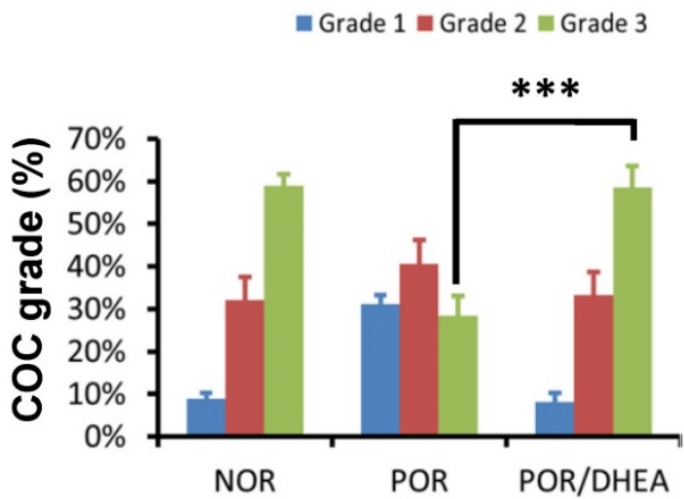

C

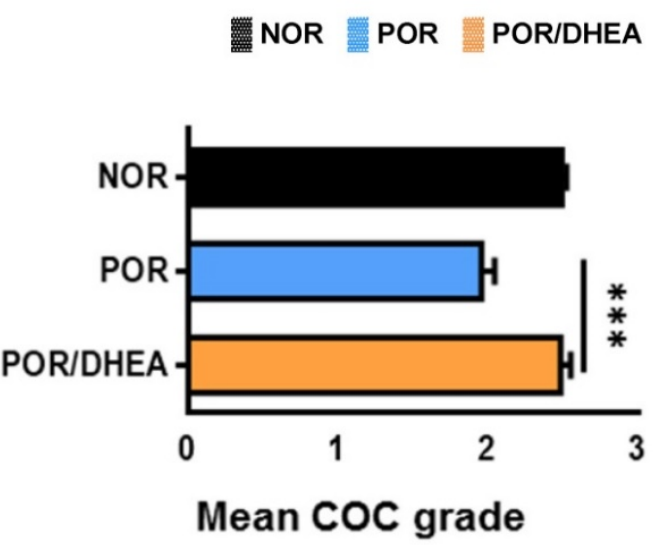

Figure 1. DHEA supplementation ameliorated cumulus-oocyte complex grade in poor ovarian responders. (A) Representative cumulus-oocyte complexes (COCs) from different groups of normal ovarian responder (NOR), poor ovarian responder (POR) and POR/DHEA were shown. (B) The COC grade was assessed among the three groups. The proportion of COC grade in each group was shown. (C) The mean COC grade was compared among the three groups. Scale bar $=25 \mu \mathrm{m}$. Data represented the mean \pm standard deviation. $* * * p<0.001$. 


\section{DHEA supplementation suppressed apoptosis in cumulus cells}

The mRNA levels of $B A X, B A D$, caspase-3, caspase-9 and cytochrome c significantly decreased in the CCs from the POR/DHEA group compared with those from the POR group (Fig. 2A). Furthermore, BCL2 mRNA was greater in the CCs from the POR/DHEA group than those from the POR group (Fig. 2A). To further confirm the anti-apoptotic effect of DHEA, TUNEL staining was performed to directly assess the percentage of apoptotic cells in the presence or absence of DHEA in the PORs. The POR/DHEA group was associated with a significantly lower percentage of apoptotic cells when compared to the POR group ( $9.7 \%$ vs. $85.7 \%$, respectively, $p<0.001$ ) (Fig. 2B). Consistent with the results observed using Q-PCR, the addition of DHEA significantly reduced apoptosis of CCs.

A
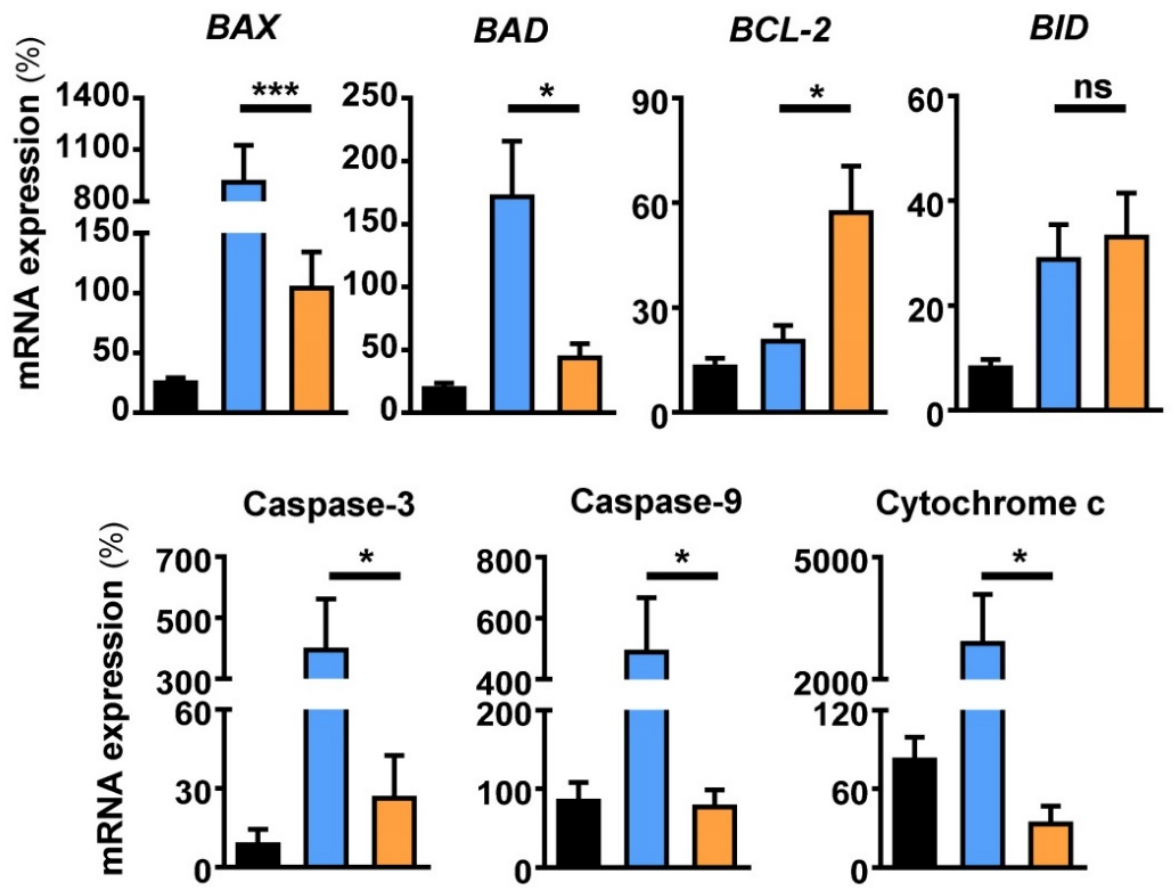

B

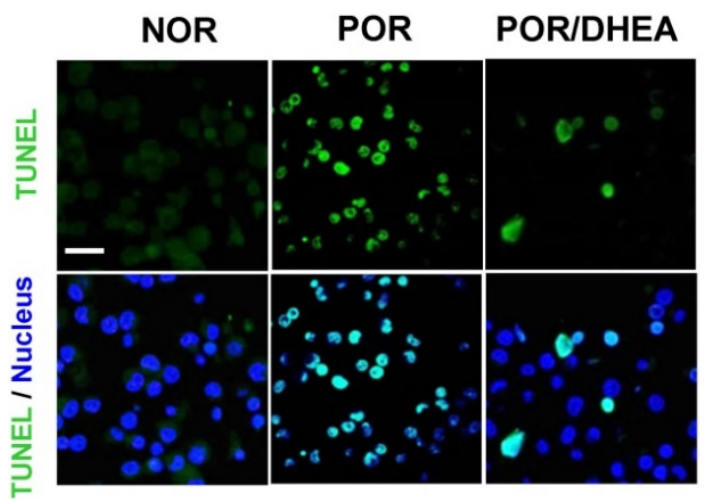

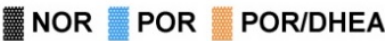

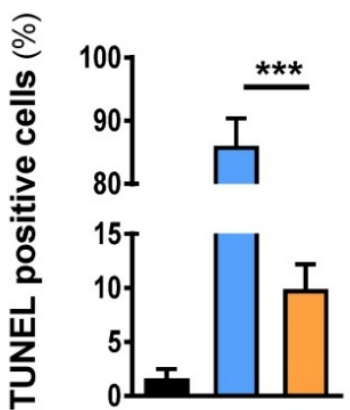

Figure 2. DHEA supplementation reduced apoptosis of cumulus cells in poor ovarian responders. (A) Quantitative real-time polymerase chain reaction analysis for mRNA expression of apoptosis-related genes of cumulus cells (CCs) among the normal ovarian responder (NOR), poor ovarian responder (POR) and POR/DHEA groups. (B) Representative confocal microscopy images of DNA fragmentation in CCs were shown. DNA fragmentation, detected by terminal deoxynucleotidyl transferase dUTP nick end labeling, was depicted by green fluorescence, and all cell nuclei, stained with 4',6-diamidino-2-phenylindole (DAPI), were depicted by blue fluorescence. Quantitative analysis of apoptotic cells in CCs among the three groups was performed. Scare bar $=20 \mu \mathrm{m}$. Data represented the mean \pm standard deviation of three independent experiments. $* p<0.05$, *** $p<0.001$; ns, non-significant. 


\section{Effects of DHEA supplementation on the mitochondria in cumulus cells}

As shown in Fig. 3, compared to the CCs from the NOR group, the $\mathrm{CCs}$ from the POR group exhibited markedly lower expression of mitochondrial transcription factor A (TFAM) gene and reduced mitochondrial dehydrogenase activity. However, DHEA supplementation significantly increased the TFAM gene expression and enhanced mitochondrial dehydrogenase activity in CCs from the POR (Fig. 3A and 3B). To further assess whether supplementation with DHEA was sufficient to improve mitochondrial function, we analyzed the mitochondrial mass using real-time image cytometry. By visualizing the mitochondria with fluorescent MitoTracker green, lower mitochondrial mass was observed in the CCs from the POR group than those from the NOR group. However, mitochondrial mass in CCs from the POR remarkably increased following DHEA supplementation (Fig. 3C).
A

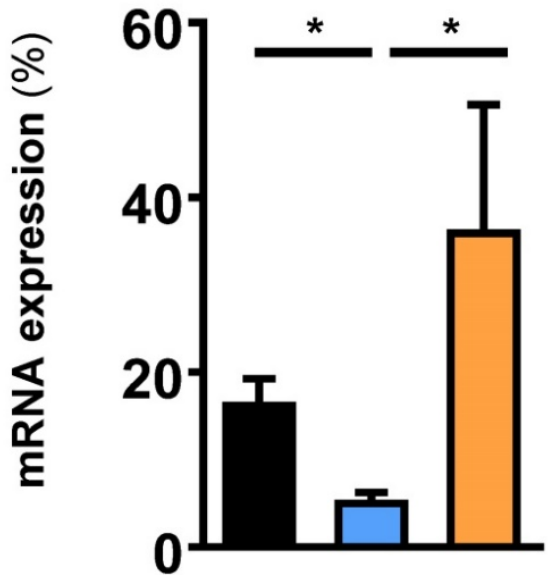

Quantitative image cytometry

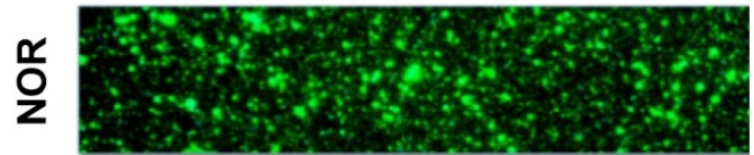

$\frac{1}{0}$

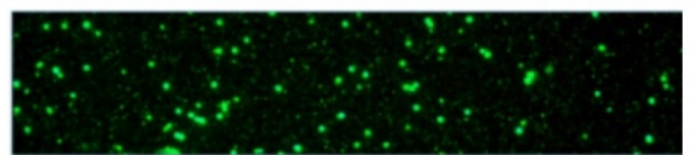

총품

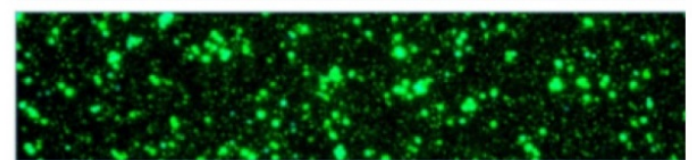

NOR

䉰 POR

POR/DHEA

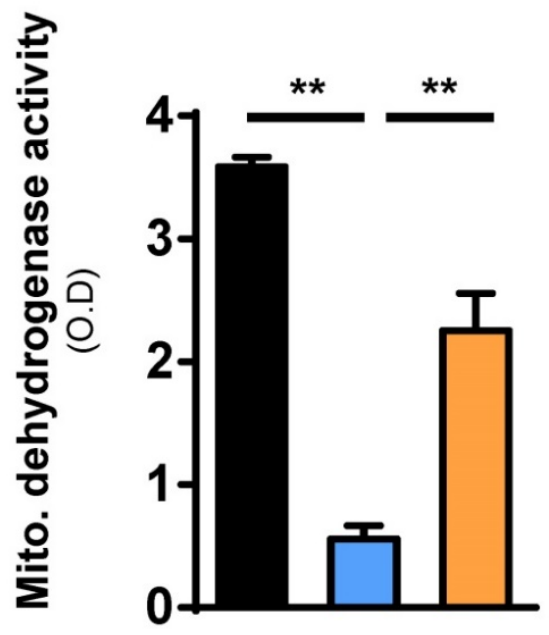

Figure 3. DHEA supplementation improved mitochondrial function of cumulus cells in poor ovarian responders. (A) Quantitative real-time polymerase chain reaction analysis for mRNA expression of TFAM gene of cumulus cells (CCs) among the normal ovarian responder (NOR), poor ovarian responder (POR) and POR/DHEA groups. (B) Mitochondrial dehydrogenase activity was assessed among the three groups. (C) CCs were stained with MitoTracker green, and the mitochondrial mass was measured by real-time image cytometry. The relative mean of fluorescent intensity (MFI) was calculated among the three groups. Data represented the mean \pm standard deviation of three independent experiments. $* p<0.05, * * p<0.01$, *** $p<0.001$. 


\section{Discussion}

This prospective cohort study demonstrated that PORs following DHEA supplementation significantly improved the proportion of grade 3 COC and mean COC grade and markedly increased the number of top-quality embryos at day 3, transferred embryos and fertilization rate compared to PORs without DHEA supplementation. Additionally, PORs with DHEA pretreatment displayed a tendency of higher clinical pregnancy rate, ongoing pregnancy rate and live birth rate than PORs without DHEA pretreatment. These results supported the beneficial effects of DHEA on IVF outcomes in the previous studies $[7,8]$. A total of three meta-analyses have revealed that pretreatment with DHEA was associated with increased pregnancy rates or live birth rates in PORs undergoing IVF cycles [11, 32, 33]. Moreover, an updated randomized controlled trial demonstrated that supplementation with DHEA in PORs significantly increased the number of retrieved oocytes, fertilized oocytes, high-quality embryos, fertilization rate and pregnancy rates [34]. Therefore, DHEA, widely used as an adjuvant to IVF cycles in PORs worldwide, has been regarded as a potential intervention to improve reproductive outcomes in PORs. However, more large-scale, well-designed randomized controlled trials are needed to verify the results.

In the current study, supplementation with DHEA significantly decreased expressions of the pro-apoptotic genes $(B A X, B A D)$ and caspase activation (cytochrome c, caspase-9 and caspase-3) and markedly increased expression of the anti-apoptotic gene (BCL2) in CCs (Fig. 2A). However, in our previous study, both $B A X$ and $B C L 2$ genes expressions in CCs were declined after DHEA supplementation [17]. One possible explanation for the different gene expression of BCL2 was that $B A X$ gene expression in CCs was severely suppressed and down to nearly zero after DHEA supplementation in our previous study [17], which might induce compensatory mechanisms to inhibit anti-apoptosis, resulting in decreased gene expression of BCL2 because apoptosis is important for normal ovarian physiology [19]. Furthermore, a significantly lower percentage of apoptotic cells were observed in the CCs from the POR/DHEA group than those form the POR group (Fig. 2B). These results suggested the anti-apoptotic effect of DHEA on the CCs and several studies using cell culture also supported that DHEA could defend against apoptosis [35-38]. The study conducted by Alexaki et al. exhibited a protective effect of DHEA in human keratinocytes against apoptosis through altered balance of BCL2 proteins
[35]. In the study of Liu et al., DHEA protected vascular endothelial cells against apoptosis by activating the Galphai-PI3K/Akt pathway and regulating antiapoptotic BCL2 expression [36]. Furthermore, Ding et al. demonstrated that DHEA inhibited $\mathrm{H}_{2} \mathrm{O}_{2}$-induced apoptosis in the Leydig cells through activation of PI3K/Akt signaling pathways [38].

In fact, numerous studies have showed that the apoptosis of CCs was involved in oocyte maturity, fertilization, embryo development and pregnancy outcome [20-23]. Host et al. observed that apoptosis in CCs was highly correlated with retarded nuclear development or atresia of the oocyte, which impaired the maturity and fertilization of the oocyte [20]. The study conducted by Corn et al. demonstrated that a high degree of apoptosis in CCs impaired blastocyst development and quality [21]. In the study of Lee et al., the incidence of CCs apoptosis was negatively associated with the number of retrieved oocytes, the embryo grade, and the pregnancy outcomes in the IVF cycles [22]. In addition, Diaz-Fontdevila et al. demonstrated that patients with lower apoptotic rates in CCs had higher good-quality embryos and a tendency of higher pregnancy rates [23].

The present study showed that the expression of TFAM gene, mitochondrial dehydrogenase activity and mitochondrial mass were higher in the CCs from the POR/DHEA group than those from the POR group. TFAM is an essential protein that binds mitochondrial DNA (mtDNA) to regulate mitochondrial transcription initiation and is also a key regulator of mtDNA copy number [39]. The abundance of mtDNA generally reflects TFAM levels [39]. The results suggested that DHEA supplementation had the positive effects on the mitochondrial function in CCs; several studies using cell culture or an animal model also supported the beneficial effects of DHEA treatment on the mitochondrial function [35, 38, 40, 41]. In a study using human keratinocytes, DHEA reversed serum deprivation-induced reduction of mitochondrial membrane potential to basal levels and conserved mitochondrial membrane integrity [35]. Furthermore, DHEA significantly increased the activities of superoxide dismutase, catalase and peroxidase, and decreased the loss of mitochondrial membrane potential and the level of reactive oxygen species in the Leydig cells [38]. Patel et al. indicated that DHEA treatment can improve oxidative energy metabolism by promoting ATPase activity and mitochondrial dehydrogenases activities in the mitochondria of rats [40, 41].

Mitochondria in oocytes or CCs participated in oocyte maturation, fertilization, embryo development 
and pregnancy outcomes [26, 42-44]. Increased abnormal mitochondrial structure and decreased expression of mitochondrial genes were observed in human unfertilized oocytes [45, 46]. The mtDNA content in oocytes was pivotal to fertilization and served as a predictor for oocyte quality [26, 27]. Moreover, Boucret et al. concluded that mitochondrial biogenesis of the CCs may be a major determinant of oocyte competence [42]. The study conducted by Ogino et al. demonstrated that mtDNA copy number in CCs can be used to predict embryo quality [43]. In the study of Tsai et al., the mitochondria DNA 4977-bp deletion in CCs was negatively correlated with pregnancy rate during IVF cycles [44].

Taken together, DHEA treatment may ameliorate IVF outcomes partially though improving mitochondrial function and reducing apoptosis in CCs. The results of this study confirmed and strengthened the conclusions of our previous work [18] by stricter study design and further experiments. However, there were still some limitations in this study. First, the population size remained small. Thus, clinical pregnancy rate, ongoing pregnancy rate and live birth rate potentially increased following DHEA supplementation in PORs. However, the difference did not reach statistical significance. Second, this study was not a randomized controlled trial. Further randomized controlled trials are required to clarify the effect of DHEA treatment. Third, the participants enrolled based on Bologna criteria might be heterogeneous.

In conclusion, the benefits of DHEA supplementation on IVF outcomes in PORs were significant, and the DHEA effects may be partially mediated by improving mitochondrial function and reducing apoptosis of CCs. Our observations may provide a reasonable rationale for clinical uses of DHEA supplementation in PORs undergoing IVF cycles to improve clinical outcomes.

\section{Abbreviations}

AFC: antral follicle counts;

AMH: anti-Müllerian hormone;

CC: cumulus cell;

CCK-8: cell counting kit-8;

COC: cumulus-oocyte complex;

COS: controlled ovarian stimulation;

DAPI: 4',6-diamidino-2-phenylindole;

DEPC: diethylpyrocarbonate;

DHEA: dehydroepiandrosterone;

GnRH: gonadotropin-releasing hormone;

ICSI: intracytoplasmic sperm injection;

IVF: in vitro fertilization;

mtDNA: mitochondrial DNA;

NOR: normal ovarian responder;
PBS: phosphate-buffered saline,

POR: poor ovarian responder;

Q-PCR: quantitative real-time polymerase chain reaction;

rFSH: recombinant follicle-stimulating hormone;

rLH: recombinant luteinizing hormone;

TFAM: mitochondrial transcription factor A;

TUNEL: terminal deoxynucleotidyl transferase dUTP nick end labeling.

\section{Supplementary Material}

Supplemental Table S1.

http://www.medsci.org/v14p0585s1.pdf

\section{Acknowledgements}

This work was generously supported by grants VGHKS105-G06-01 from Kaohsiung Veterans General Hospital.

\section{Author Contributions}

L.T.L. and C.J.L. are responsible for performing experiments and drafting the article. P.H.W. and Z.H.W. are responsible for design of the study. S.N.C. and E.M.T. are responsible for analysis and interpretation of data. J.T.C. and K.H.T. are responsible for supervising the research and revising the manuscript. All authors reviewed the final version of the manuscript.

\section{Competing Interests}

The authors have declared that no competing interest exists.

\section{References}

1. Polyzos NP, Nwoye M, Corona R, Blockeel C, Stoop D, Haentjens P, et al. Live birth rates in Bologna poor responders treated with ovarian stimulation for IVF/ICSI. Reproductive biomedicine online. 2014; 28: 469-74.

2. Pilehvari S, ShahrokhTehraninejad E, Hosseinrashidi B, Keikhah F, Haghollahi F, Aziminekoo E. Comparison Pregnancy Outcomes Between Minimal Stimulation Protocol and Conventional GnRH Antagonist Protocols in Poor Ovarian Responders. Journal of family \& reproductive health. 2016; 10: 35-42.

3. Sunkara SK, Coomarasamy A, Faris R, Braude P, Khalaf Y. Long gonadotropin-releasing hormone agonist versus short agonist versus antagonist regimens in poor responders undergoing in vitro fertilization: a randomized controlled trial. Fertility and sterility. 2014; 101: 147-53.

4. Luo S, Li S, Li X, Qin L, Jin S. Effect of pretreatment with transdermal testosterone on poor ovarian responders undergoing IVF/ICSI: A meta-analysis. Experimental and therapeutic medicine. 2014; 8: 187-94.

5. Bosdou JK, Venetis CA, Kolibianakis EM, Toulis KA, Goulis DG, Zepiridis L, et al. The use of androgens or androgen-modulating agents in poor responders undergoing in vitro fertilization: a systematic review and meta-analysis. Human reproduction update. 2012; 18: 127-45.

6. Papaleo E, Vanni VS, Vigano P, La Marca A, Pagliardini L, Vitrano R, et al. Recombinant LH administration in subsequent cycle after "unexpected" poor response to recombinant FSH monotherapy. Gynecological endocrinology : the official journal of the International Society of Gynecological Endocrinology. 2014; 30: 813-6.

7. Lin LT, Tsui KH, Wang PH. Clinical application of dehydroepiandrosterone in reproduction: A review of the evidence. Journal of the Chinese Medical Association : JCMA. 2015; 78: 446-53.

8. Zhang J, Qiu X, Gui Y, Xu Y, Li D, Wang L. Dehydroepiandrosterone improves the ovarian reserve of women with diminished ovarian reserve and is a potential regulator of the immune response in the ovaries. Bioscience trends. 2016; 9: 350-9. 
9. Labrie F. All sex steroids are made intracellularly in peripheral tissues by the mechanisms of intracrinology after menopause. The Journal of steroid biochemistry and molecular biology. 2015; 145: 133-8.

10. Chimote NM, Nath NM, Chimote NN, Chimote BN. Follicular fluid dehydroepiandrosterone sulfate is a credible marker of oocyte maturity and pregnancy outcome in conventional in vitro fertilization cycles. Journal of human reproductive sciences. 2015; 8: 209-13.

11. Nagels HE, Rishworth JR, Siristatidis CS, Kroon B. Androgens (dehydroepiandrosterone or testosterone) for women undergoing assisted reproduction. The Cochrane database of systematic reviews. 2015; 11: Cd009749.

12. Fragouli E, Lalioti MD, Wells D. The transcriptome of follicular cells: biological insights and clinical implications for the treatment of infertility. Human reproduction update. 2014; 20: 1-11.

13. Albertini DF, Combelles CM, Benecchi E, Carabatsos MJ. Cellular basis for paracrine regulation of ovarian follicle development. Reproduction. 2001; 121: 647-53.

14. Assou S, Haouzi D, De Vos J, Hamamah S. Human cumulus cells as biomarkers for embryo and pregnancy outcomes. Molecular human reproduction. 2010; 16: 531-8.

15. Wathlet $\mathrm{S}$, Adriaenssens $\mathrm{T}$, Segers I, Verheyen $\mathrm{G}$, Janssens R, Coucke W, et al. New candidate genes to predict pregnancy outcome in single embryo transfer cycles when using cumulus cell gene expression. Fertility and sterility. 2012; 98: 432-9 e1-4.

16. Uyar A, Torrealday S, Seli E. Cumulus and granulosa cell markers of oocyte and embryo quality. Fertility and sterility. 2013; 99: 979-97.

17. Tsui KH, Lin LT, Horng HC, Chang R, Huang BS, Cheng JT, et al. Gene expression of cumulus cells in women with poor ovarian response after dehydroepiandrosterone supplementation. Taiwan J Obstet Gynecol. 2014; 53: 559-65.

18. Lin LT, Wang PH, Chen SN, Li CJ, Wen $\mathrm{ZH}$, Cheng JT, et al Protection of cumulus cells following dehydroepiandrosterone supplementation. Gynecological endocrinology : the official journal of the International Society of Gynecological Endocrinology. 2017; 33: 100-4.

19. Hussein MR. Apoptosis in the ovary: molecular mechanisms. Human reproduction update. 2005; 11: 162-77.

20. Host E, Gabrielsen A, Lindenberg S, Smidt-Jensen S. Apoptosis in human cumulus cells in relation to zona pellucida thickness variation, maturation stage, and cleavage of the corresponding oocyte after intracytoplasmic sperm injection. Fertility and sterility. 2002; 77: 511-5.

21. Corn CM, Hauser-Kronberger C, Moser M, Tews G, Ebner T. Predictive value of cumulus cell apoptosis with regard to blastocyst development of corresponding gametes. Fertility and sterility. 2005; 84: 627-33.

22. Lee KS, Joo BS, Na YJ, Yoon MS, Choi OH, Kim WW. Cumulus cells apoptosis as an indicator to predict the quality of oocytes and the outcome of IVF-ET. Journal of assisted reproduction and genetics. 2001; 18: 490-8.

23. Diaz-Fontdevila M, Pommer R, Smith R. Cumulus cell apoptosis changes with exposure to spermatozoa and pathologies involved in infertility. Fertility and sterility. 2009; 91: 2061-8.

24. Xiong S, Mu T, Wang G, Jiang X. Mitochondria-mediated apoptosis in mammals. Protein \& cell. 2014; 5: 737-49.

25. Roy MI, Vom A, Czabotar PE, Lessene G. Cell death and the mitochondria: therapeutic targeting of the BCL-2 family-driven pathway. British journal of pharmacology. 2014; 171: 1973-87.

26. Santos TA, El Shourbagy S, St John JC. Mitochondrial content reflects oocyte variability and fertilization outcome. Fertility and sterility. 2006; 85: 584-91.

27. Zeng HT, Ren Z, Yeung WS, Shu YM, Xu YW, Zhuang GL, et al. Low mitochondrial DNA and ATP contents contribute to the absence of birefringent spindle imaged with PolScope in in vitro matured human oocytes. Human reproduction. 2007; 22: 1681-6.

28. Lee SK, Zhao MH, Kwon JW, Li YH, Lin ZL, Jin YX, et al. The association of mitochondrial potential and copy number with pig oocyte maturation and developmental potential. The Journal of reproduction and development. 2014; 60: 128-35.

29. Ferraretti AP, La Marca A, Fauser BC, Tarlatzis B, Nargund G, Gianaroli L, et al. ESHRE consensus on the definition of 'poor response' to ovarian stimulation for in vitro fertilization: the Bologna criteria. Human reproduction. 2011; 26: 1616-24.

30. The Istanbul consensus workshop on embryo assessment: proceedings of an expert meeting. Human reproduction. 2011; 26: 1270-83.

31. Veeck LL, Wortham JW, Jr., Witmyer J, Sandow BA, Acosta AA, Garcia JE, et al. Maturation and fertilization of morphologically immature human oocytes in a program of in vitro fertilization. Fertility and sterility. 1983; 39: 594-602.

32. Li J, Yuan $\mathrm{H}$, Chen $\mathrm{Y}, \mathrm{Wu} \mathrm{H}, \mathrm{Wu} \mathrm{H}, \mathrm{Li} \mathrm{L}$. A meta-analysis of dehydroepiandrosterone supplementation among women with diminished ovarian reserve undergoing in vitro fertilization or intracytoplasmic sperm injection. International journal of gynaecology and obstetrics: the official organ of the International Federation of Gynaecology and Obstetrics. 2015; 131: $240-5$.

33. Zhang $\mathrm{M}$, Niu $\mathrm{W}$, Wang $\mathrm{Y}, \mathrm{Xu}$ J, Bao $\mathrm{X}$, Wang $\mathrm{L}$, et al. Dehydroepiandrosterone treatment in women with poor ovarian response undergoing IVF or ICSI: a systematic review and meta-analysis. Journal of assisted reproduction and genetics. 2016; 33: 981-91.

34. Kotb MM, Hassan AM, AwadAllah AM. Does dehydroepiandrosterone improve pregnancy rate in women undergoing IVF/ICSI with expected poor ovarian response according to the Bologna criteria? A randomized controlled trial. European journal of obstetrics, gynecology, and reproductive biology. 2016; 200: 11-5.

35. Alexaki VI, Charalampopoulos I, Panayotopoulou M, Kampa M, Gravanis A Castanas E. Dehydroepiandrosterone protects human keratinocytes against apoptosis through membrane binding sites. Experimental cell research. 2009; 315: 2275-83.

36. Liu D, Si H, Reynolds KA, Zhen W, Jia Z, Dillon JS. Dehydroepiandrosterone protects vascular endothelial cells against apoptosis through a Galphai protein-dependent activation of phosphatidylinositol 3-kinase/Akt and regulation of antiapoptotic Bcl-2 expression. Endocrinology. 2007; 148: 3068-76.

37. Charalampopoulos I, Tsatsanis C, Dermitzaki E, Alexaki VI, Castanas E, Margioris AN, et al. Dehydroepiandrosterone and allopregnanolone protect sympathoadrenal medulla cells against apoptosis via antiapoptotic Bcl-2 proteins. Proc Natl Acad Sci U S A. 2004; 101: 8209-14.

38. Ding $\mathrm{X}$, Wang $\mathrm{D}, \mathrm{Li} \mathrm{L}, \mathrm{Ma} \mathrm{H}$. Dehydroepiandrosterone ameliorates H2O2-induced Leydig cells oxidation damage and apoptosis through inhibition of ROS production and activation of PI3K/Akt pathways. The international journal of biochemistry \& cell biology. 2016; 70: 126-39.

39. Campbell CT, Kolesar JE, Kaufman BA. Mitochondrial transcription factor A regulates mitochondrial transcription initiation, DNA packaging, and genome copy number. Biochim Biophys Acta. 2012; 1819: 921-9.

40. Patel MA, Katyare SS. Effect of dehydroepiandrosterone (DHEA) treatment on oxidative energy metabolism in rat liver and brain mitochondria. A dose-response study. Clinical biochemistry. 2007; 40: 57-65.

41. Patel MA, Katyare SS. Treatment with dehydroepiandrosterone (DHEA) stimulates oxidative energy metabolism in the cerebral mitochondria. A comparative study of effects in old and young adult rats. Neuroscience letters. 2006; 402: 131-6.

42. Boucret L, Chao de la Barca JM, Moriniere C, Desquiret V, Ferre-L'Hotellier V, Descamps $\mathrm{P}$, et al. Relationship between diminished ovarian reserve and mitochondrial biogenesis in cumulus cells. Human reproduction. 2015; 30: 1653-64

43. Ogino $\mathrm{M}$, Tsubamoto $\mathrm{H}$, Sakata $\mathrm{K}$, Oohama N, Hayakawa $\mathrm{H}$, Kojima T, et al. Mitochondrial DNA copy number in cumulus cells is a strong predictor of obtaining good-quality embryos after IVF. Journal of assisted reproduction and genetics. 2016; 33: 367-71

44. Tsai HD, Hsieh YY, Hsieh JN, Chang CC, Yang CY, Yang JG, et al. Mitochondria DNA deletion and copy numbers of cumulus cells associated with in vitro fertilization outcomes. The Journal of reproductive medicine. 2010; 55: 491-7.

45. Au HK, Yeh TS, Kao SH, Tzeng CR, Hsieh RH. Abnormal mitochondrial structure in human unfertilized oocytes and arrested embryos. Ann N Y Acad Sci. 2005; 1042: 177-85.

46. Hsieh RH, Au HK, Yeh TS, Chang SJ, Cheng YF, Tzeng CR. Decreased expression of mitochondrial genes in human unfertilized oocytes and arrested embryos. Fertility and sterility. 2004; 81 Suppl 1: 912-8. 